\section{Recanalized Image of a Thrombotic Occlusion: A Lotus Root-Like Panda Face Appearance by Optical Coherence Tomography}

\section{Abstract}

In OCT, images of recanalized thrombotic occlusion have a lotus root-like appearance. This is due to septa that divide the lumen into multiple channels that communicate together and converge into a single lumen in the proximal and distal sites of the occlusion. We report a case of a recanalized mid left anterior descending (LAD) occlusion confirmed by OCT, where the lotus root appearance has a delightful panda face.

Keywords: Lotus root, Coronary artery, Optical coherence tomography

\section{Michaela B Rehman, Elisa Larrieu-Ardillouze and Sébastien Levesque}

CHU de Poitiers, 2 Rue de la milétrie, France

Corresponding author: Sébastien Levesque

झ selevesq@yahoo.fr

DMC Cardiologie, CHU Poitiers, 2 rue de la milétrie 86000 Poitiers, France.

Tel: 0549444628

Citation: Rehman MB, Larrieu-Ardilouze $E$, Levesque S. Recanalized Image of a Thrombotic Occlusion: A Lotus RootLike Panda Face Appearance by Optical Coherence Tomography. Interv Cardiol J 2015, 2:1.

where the lotus root appearance had a delightful panda face! The occlusion was treated with a drug eluting stent with an excellent angiographic result.

It is amusing to show how serious pathology can smile as us through this adorable face.

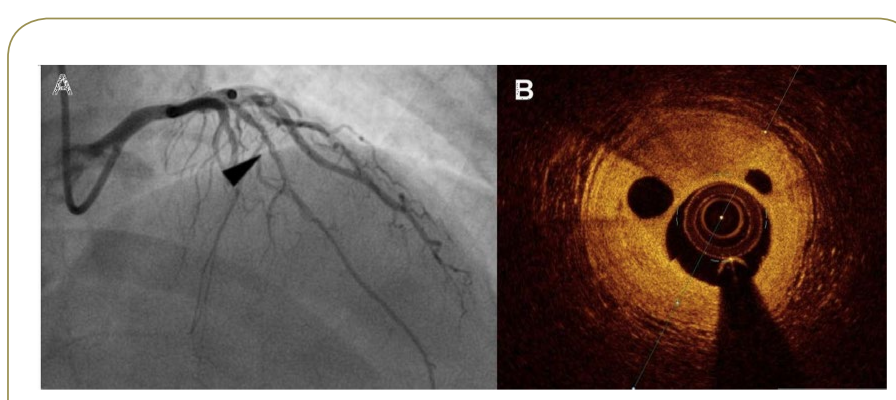

Figure 1 A) Angiogram showing the recanalized occlusion of the mid LAD (black narrow) B) OCT Axial view of the recanalized occlusion "Lotus root / Panda's Face". 


\section{References}

1 Kato M, Dote K, Sasaki S (2011) Recanalized image of thrombotic occlusion with coronary plaque rupture: a lotus root-like appearance by optical coherence tomography. Canadian Journal of Cardiology 27: 871.
2 Sakurai S, Takashima H, Waseda K, Ando H, Kurita A, et al. (2014) Multiple recanalized images of thrombotic occlusion 19 years after percutaneous coronary intervention: Insights from optical coherence tomography and intravascular ultrasound. International journal of cardiology 172: 480-481. 